\title{
Klippel-Feil syndrome with multiple skeletal anomalies, Dandy-Walker spectrum, and occipital cephalocele-a rare presentation
}

\author{
Mohammad Shoaib ${ }^{*}$ (D) M. Sarthak Swarup and Gaurav Pradhan
}

\begin{abstract}
Background: Klippel-Feil syndrome (KFS) is a congenital malformation causing fusion of at least two cervical vertebrae and characterized clinically by presence of triad of short neck, limited neck movements, and low posterior hair line. Various skeletal and non-skeletal anomalies may be seen in association with KFS.

Case presentation: We present a case of 6-year-old boy of KFS with various skeletal anomalies such as multiple segmentation and formation anomalies of the spine including anomalies of craniovertebral junction (CVJ), spina bifida occulta, scoliosis, Sprengel deformity of right shoulder, and multiple rib anomalies, as well as neurological anomalies like Dandy-Walker spectrum and atretic occipital cephalocele.

Conclusion: To the best of our knowledge, association of such extensive skeletal anomalies and Dandy-Walker spectrum with KFS has been uncommonly reported in the literature. This case highlights the importance of knowledge of various common and uncommon associations of KFS to avoid missing significant anomalies.
\end{abstract}

Keywords: Klippel-Feil syndrome, Sprengel deformity, Spina bifida, Dandy-Walker, Cephalocele, Craniovertebral junction, Scoliosis, Omovertebral bone

\section{Background}

Klippel-Feil syndrome (KFS) is described as a congenital fusion of at least two cervical vertebrae and is often characterized clinically by presence of triad of short neck, limited neck movements, and low posterior hair line. It occurs due to the failure of the segmentation of mesodermal somites during the 3rd to 8th week of intrauterine life [1]. The exact etiology is unclear; however, certain genes such as GDF6, GDF3, MEOX1, and RIPP LY2 responsible for regulation of transcription and signaling pathways of somite development are thought to be causative in the pathogenesis [2]. The estimated incidence is 1 in 40,000-42,000 with a female predilection [2]. The disorder does not just show fused cervical vertebrae but can have multi-system involvement presenting as multiple skeletal and non-skeletal anomalies. The

\footnotetext{
* Correspondence: shoaibansari28@gmail.com

Department of Radiodiagnosis, Maulana Azad Medical College and Lok Nayak Hospital, Bahadur Shah Zafar Marg, New Delhi 110002, India
}

radiologist helps to not only confirm the clinical diagnosis but has a much larger role in the demonstration of a multitude of associated anomalies which have prognostic significance. Our case is unique due to the presence of multiple skeletal anomalies such as multiple segmentation and formation anomalies of the spine, craniovertebral junction (CVJ) anomalies, Sprengel deformity, multiple rib anomalies, and non-skeletal anomalies such as Dandy-Walker spectrum and atretic cephalocele in the same patient, which to the best of our knowledge is uncommonly reported in the literature.

\section{Case presentation}

A 6-year-old boy presented to our institute with complaints of restricted neck movements, deviation of the neck towards the right side, restricted range of motion at right shoulder joint, and deformity of thoracolumbar spine since birth. The patient was one of the three children of a couple from non-consanguineous marriage. In 
antenatal history, the mother did not receive any antenatal care, and the child was born via normal vaginal delivery at home. There was no family history of spinal or neural anomalies (Figs. 1, 2, 3, 4, 5, 6, and 7).

Physical examination demonstrated short neck, low posterior hairline, torticollis with a head tilt towards the right side and contralateral chin lift, elevated right scapula, visible scoliotic deformity of the thoracolumbar spine with convexity towards the left side. There was also a soft tissue swelling in the occipital region of the scalp.

The patient was referred to our department for radiographs of chest and spine which revealed elevated right scapula (Rigault's classification grade II) and right-sided hemivertebrae at D9 and L5 with resultant scoliotic deformity with convexity towards the left side. Further imaging evaluation with non-contrast computed tomography (NCCT) was advised for better characterization of vertebral anomalies and other associated skeletal deformities. Magnetic resonance imaging (MRI) of brain and whole spine was also performed for characterization of scalp swelling and to detect any associated central nervous system (CNS) anomalies. Abdominal ultrasonography and echocardiography were done to rule out renal and cardiac anomalies respectively which were normal.

NCCT of the spine revealed scoliotic deformity of the spine with convexity towards the left side in the dorso lumbar spine and convexity towards the right side in the cervico dorsal spine. Multiple skeletal anomalies which were not appreciable on plain radiographs were also detected. These included CVI anomalies (hypoplastic right occipital condyle, type A defect of the posterior arch of atlas and anterior arch cleft, hypoplastic odontoid process with atlantoaxial instability, and spinal canal stenosis), multiple segmentation and formation anomalies of the spine (fused posterior elements of C4-C5 and C5-C6; non-fusion of posterior elements of C4, C5, C6, $\mathrm{C} 7$, and L5 (spina bifida occulta); right-sided hemivertebrae at D1, D9, and L5 with hypoplastic left-sided posterior elements of D9 and L5). Right scapula and right clavicle were elevated lying between C5 and D4 levels with a thick bony bar seen arising from the fused rightsided neural arch of C5-C6 and extending posterolaterally towards superomedial part of elevated scapula (omovertebral bone). Multiple rib anomalies were also observed which included right-sided cervical rib, bifid left-sided first rib, fused left-sided second and third ribs near vertebral attachment, absent left 9th rib, and bilateral 12th ribs.

MRI brain revealed isolated inferior vermian hypoplasia with a cerebrospinal fluid (CSF)-filled cystic structure communicating with the fourth ventricle in posterior fossa. The findings were consistent with Dandy-Walker spectrum. There was also the presence of an occipital scalp swelling with areas of CSF signal intensity, which is seen to communicate with enlarged posterior fossa cyst through a calvarial defect. There was also associated vertical orientation of straight sinus. These findings were consistent with atretic cephalocele. MRI spine revealed kinking of cervicomedullary junction, obliteration of both anterior, and posterior subarachnoid spaces; however, normal signal intensity is noted within the spinal cord. USG abdomen did not reveal any abnormalities.
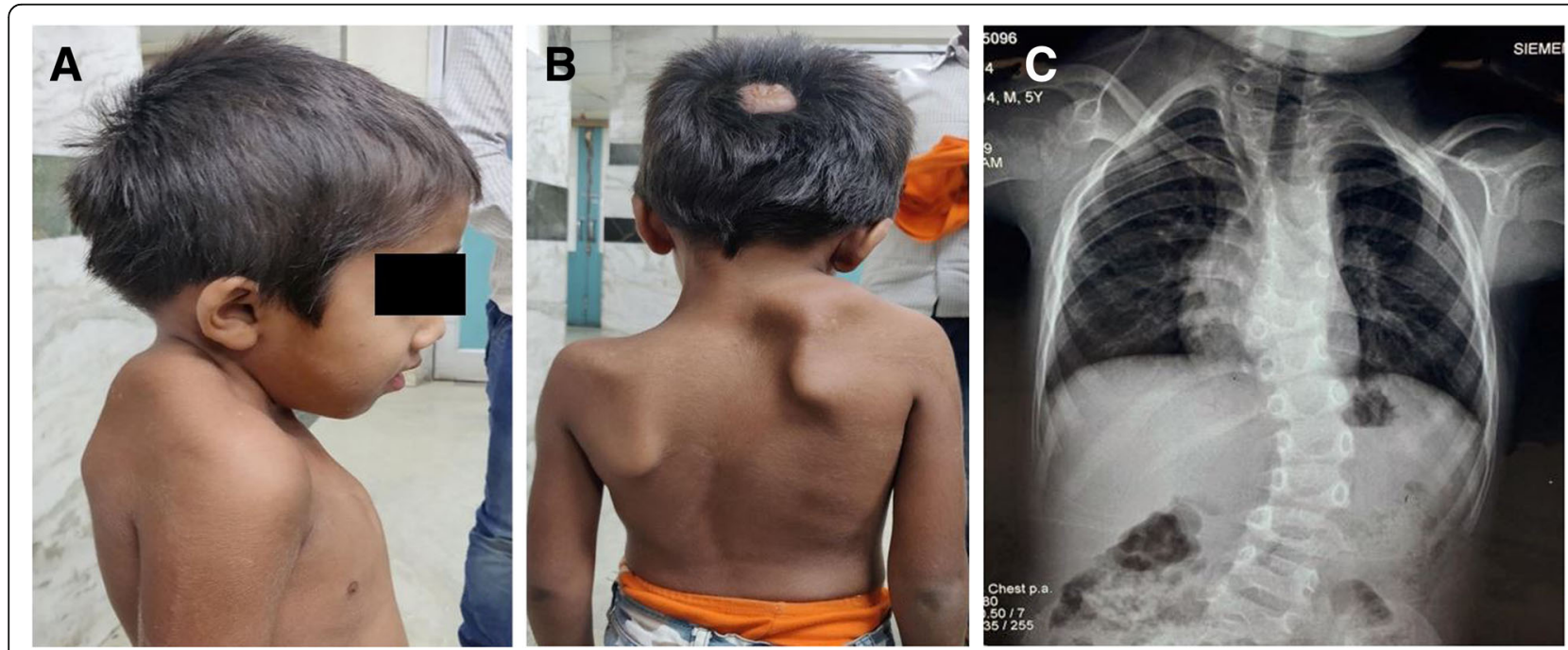

Fig. 1 a Photographs of a 6-year-old patient with Klippel-Feil syndrome and Sprengel deformity showing the clinical profile of the patient showing short neck and elevation of right scapula. $\mathbf{b}$ There is also torticollis with head tilt towards right side, visible scoliotic deformity of thoracolumbar spine and a scalp swelling. c AP radiograph of chest and abdomen shows Sprengel deformity with hemivertebra at D1, D9, and L5 and resultant scoliotic deformity 

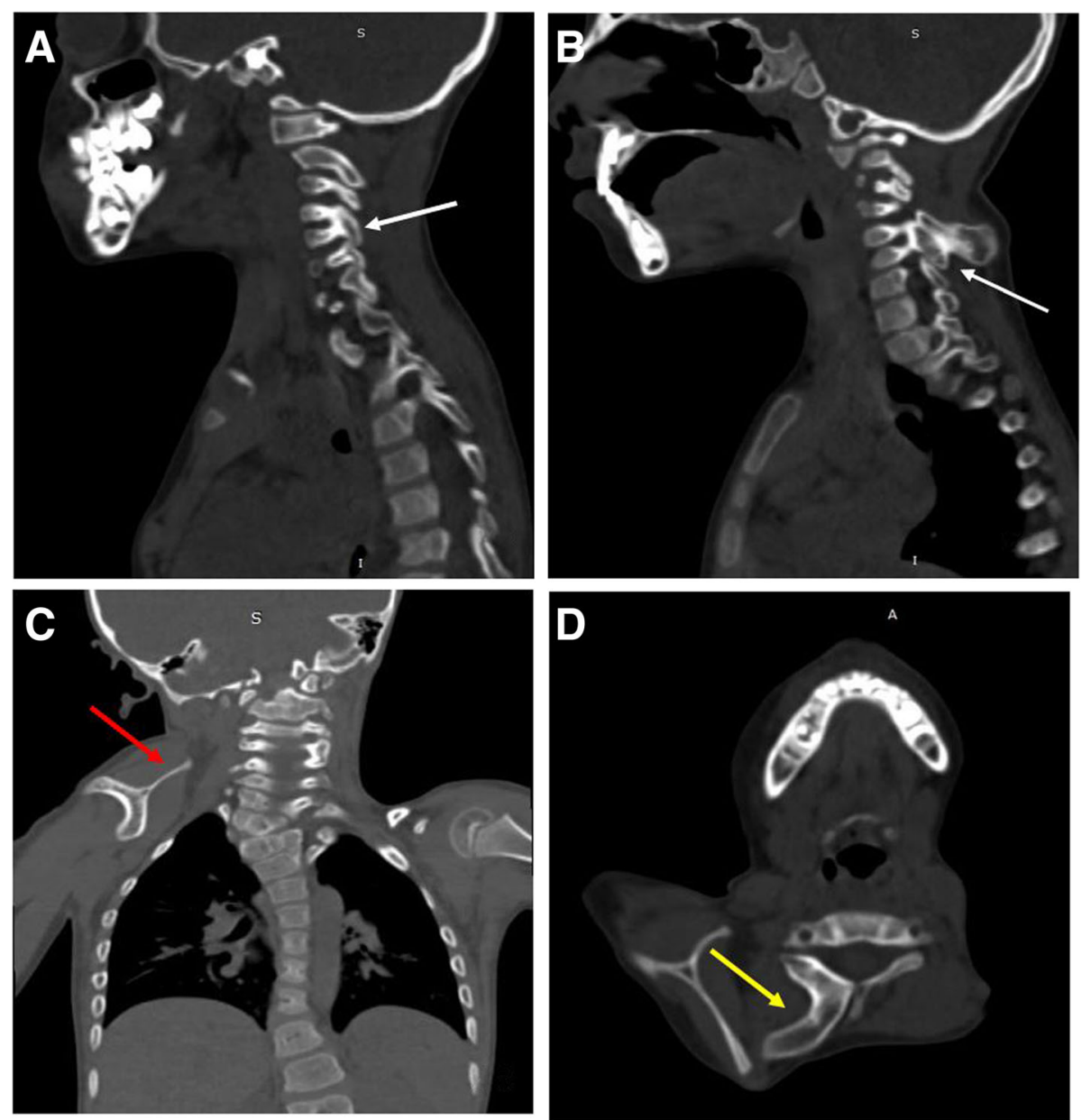

Fig. 2 a Sagittal reformatted non-contrast CT image of the cervical spine shows fusion of posterior elements of C4-C5 (white arrow). b Sagittal reformatted non-contrast CT image of the cervical spine shows fusion of posterior elements of C5-C6 (white arrow). c Coronal reformatted CT image shows elevated right scapula with its superomedial angle lying between C5 and D2 vertebral levels suggestive of grade II Sprengel deformity (red arrow). d Axial bone window CT image shows a thick bony bar arising from fused C5-C6 neural arch and reaching up to the superomedial angle of scapula suggestive of omovertebral bone (yellow arrow)

Echocardiography was also normal. The patient was being considered for genetic testing for genes associated with KFS; however, due to financial constraints of the patient, it could not be done.

The patient was operated for Sprengel deformity following which he had significant improvement in range of motion at the shoulder joint. The parents were explained about the prognosis of associated anomalies and are put on follow-up for further management of scoliosis and planned excision of atretic cephalocele.

\section{Discussion}

In the year 1912, Maurice Klippel first described KlippelFeil syndrome (KFS) as a congenital malformation causing fusion of cervical vertebrae which can involve either two vertebrae or entire cervical spine. In the same year, Feil classified KFS into 3 types based on the degree of involvement as type I (massive fusion of cervical and upper thoracic vertebrae), type II (fusion of 2 or more vertebrae with associated atlanto-occipital assimilation, hemivertebrae, or any other anomaly of the cervical spine), and type III (fused cervical vertebrae with lower thoracic or lumbar vertebrae). Diagnosis can be suggested by classical findings described as a triad of restricted neck movements, short neck, and low posterior hairline; however, complete triad is seen in only about $50 \%$ of the patients [3].

Various osseous and non-osseous anomalies are associated with KFS, the most common being scoliosis seen in $70 \%$ of cases [4]. Other commonly associated skeletal anomalies include Sprengel deformity (20-30\%), spina bifida occulta (45\%), and craniovertebral junction anomalies; all of these were seen in our patient [5]. Due to fusion anomalies (fused posterior elements of C4-C5 and C5-C6 vertebrae) and multiple hemivertebrae at D1, D9, and L5 seen in the patient, our patient fit the criteria for type II KFS [3]. 

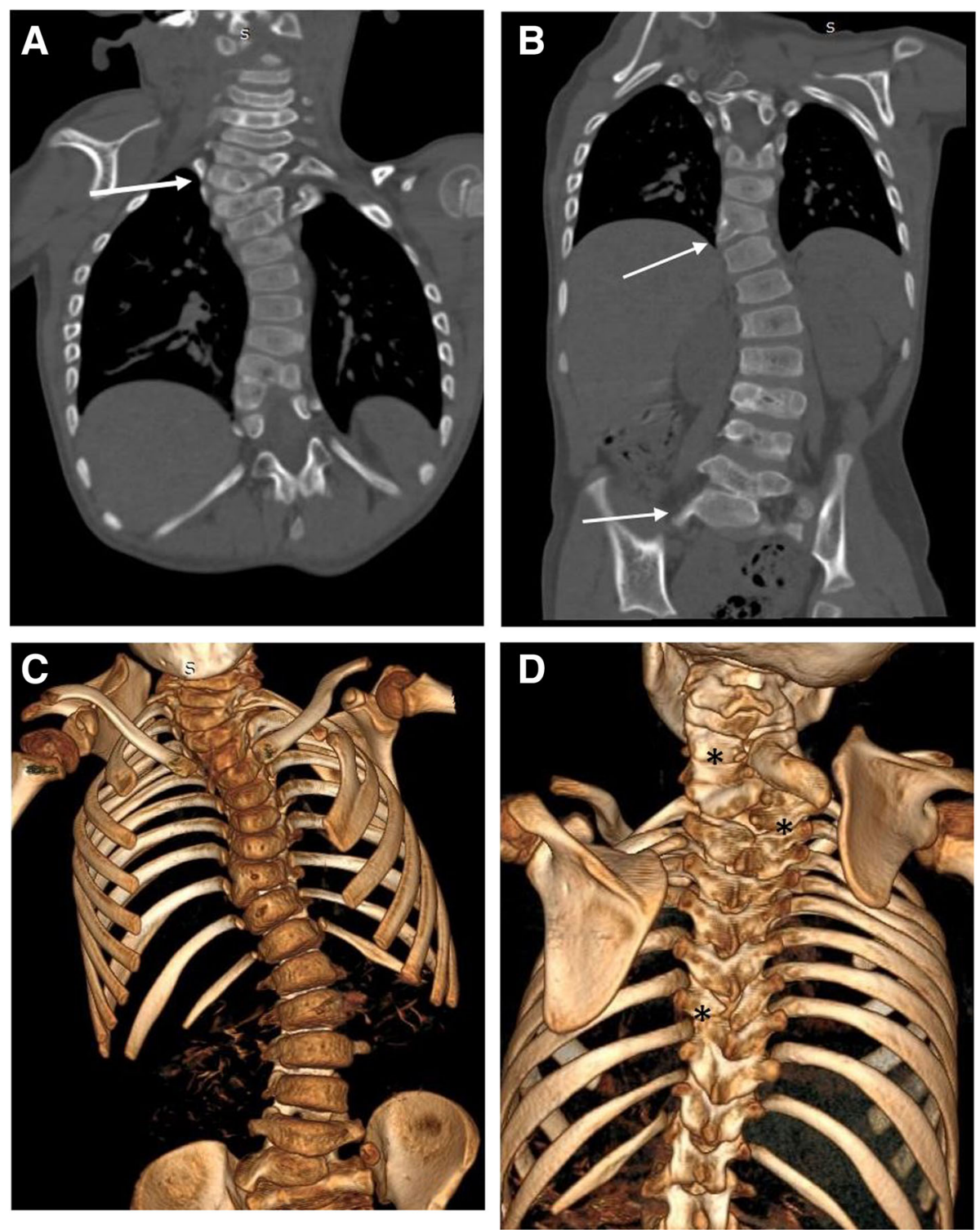

Fig. 3 a Coronal reformatted images of the cervicodorsal spine show hemivertebra at D1 level (white arrow). b Coronal reformatted images of the cervicodorsal spine show hemivertebra at D9 and L5 levels (white arrows). c Three-dimensional volume rendered CT images of the cervico dorsal spine show hemivertebrae at D1, D9, and L5 levels. $\mathbf{d}$ Three-dimensional volume rendered CT images of the cervico dorsal spine show non-fusion of posterior elements of C4-C6 and D4 vertebrae (black asterisks).

Sprengel deformity is classified radiographically into 3 grades based on the position of the superomedial angle of scapula, given by Rigualt [6]. This includes grade I (between D2 and D4 transverse processes), grade II (between $\mathrm{C} 5$ and D2 transverse processes), and grade III (above C5 transverse process). Our patient belonged to grade II Sprengel deformity. Sprengel deformity is often fixed with adjacent vertebrae by presence of omovertebral bone which limits the necessary scapular rotation needed for overhead arm abduction. Omovertebral bone was seen in our patient extending from neural arch of C5-C6 to superomedial part of elevated scapula. The patient underwent resection of omovertebral bone following which he gained a significant range of motion at shoulder which was previously limited.

Among other skeletal anomalies, our patient also had multiple rib anomalies which included cervical rib, adjacent fused ribs, bifid ribs, and absent ribs. Rib anomalies are seen rarely in association with KFS with few case reports in the literature.

Apart from skeletal anomalies, associated common visceral anomalies in KFS include renal anomalies which usually occur in the form of unilateral agenesis, CNS abnormalities such as meningocele, syringomyelia, 

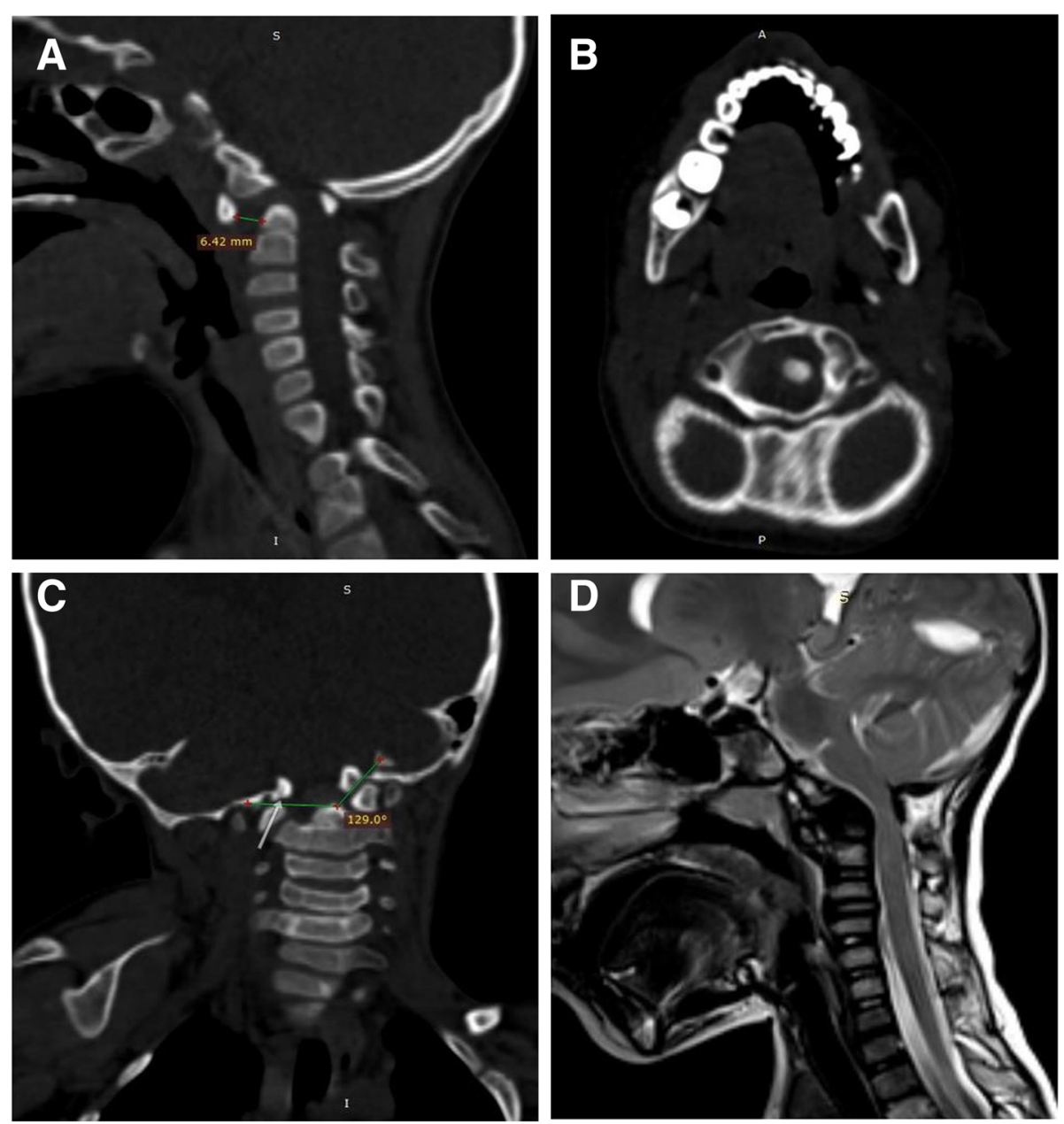

Fig. 4 a Non-contrast sagittal reformatted CT image shows hypoplastic odontoid process with associated atlantoaxial instability and increased atlanto-dens interval. b Axial non-contrast CT image shows and anterior arch cleft and midline cleft in the posterior arch of atlas suggestive of type A defect. c Coronal reformatted CT image shows hypoplasia of right occipital condyle (white arrow) with widened Schmidt Fischer angle. $\mathbf{d}$ Sagittal T2- weighted MRI image of the same patient showing kinking of cervicomedullary junction with obliteration of anterior and posterior subarachnoid spaces
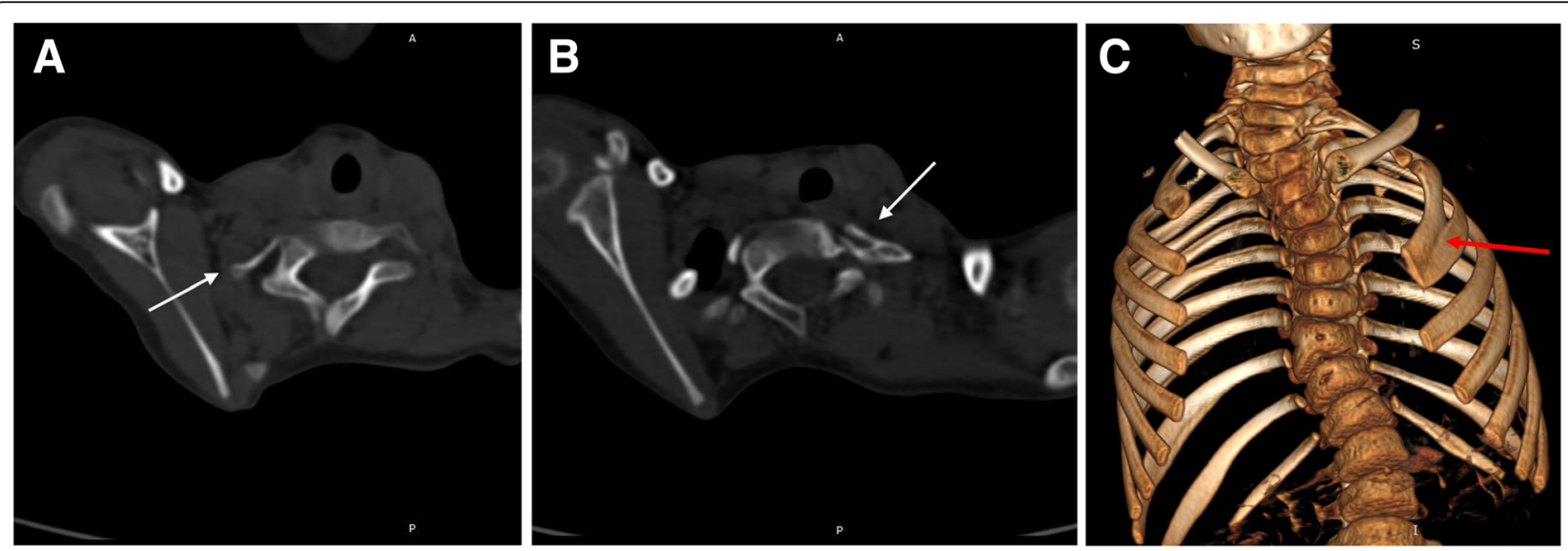

Fig. 5 a Axial non-contrast CT image show presence of hypoplastic right cervical rib (white arrow). b Axial non contrast CT image show presence of bifid left-sided 1st rib (white arrow). c Volume rendered three-dimensional CT images of the same patient demonstrating fused left 2 nd and 3rd ribs (red arrow) and presence of aplastic left 9th rib and bilateral 12th ribs 

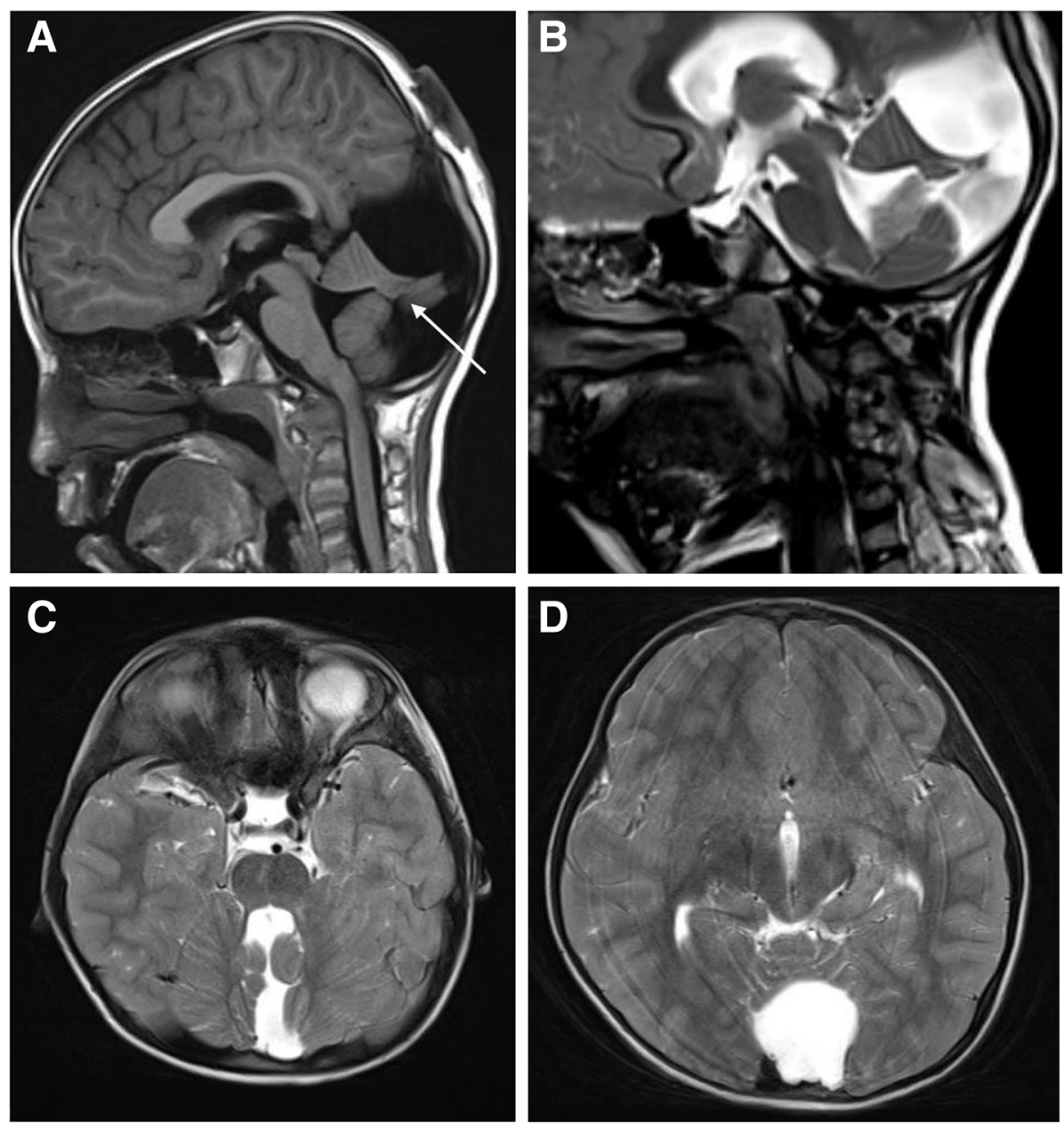

Fig. $\mathbf{6}$ a, b Sagittal T1-weighted and T2-weighted MR images of the brain demonstrate Dandy-Walker spectrum with isolated inferior vermian hypoplasia with counter clock wise rotation of superior vermian remnant (white arrow). $\mathbf{c}, \mathbf{d}$ Axial T2 weighted images of the same patient demonstrate a posterior fossa cyst communicating with the 4th ventricle
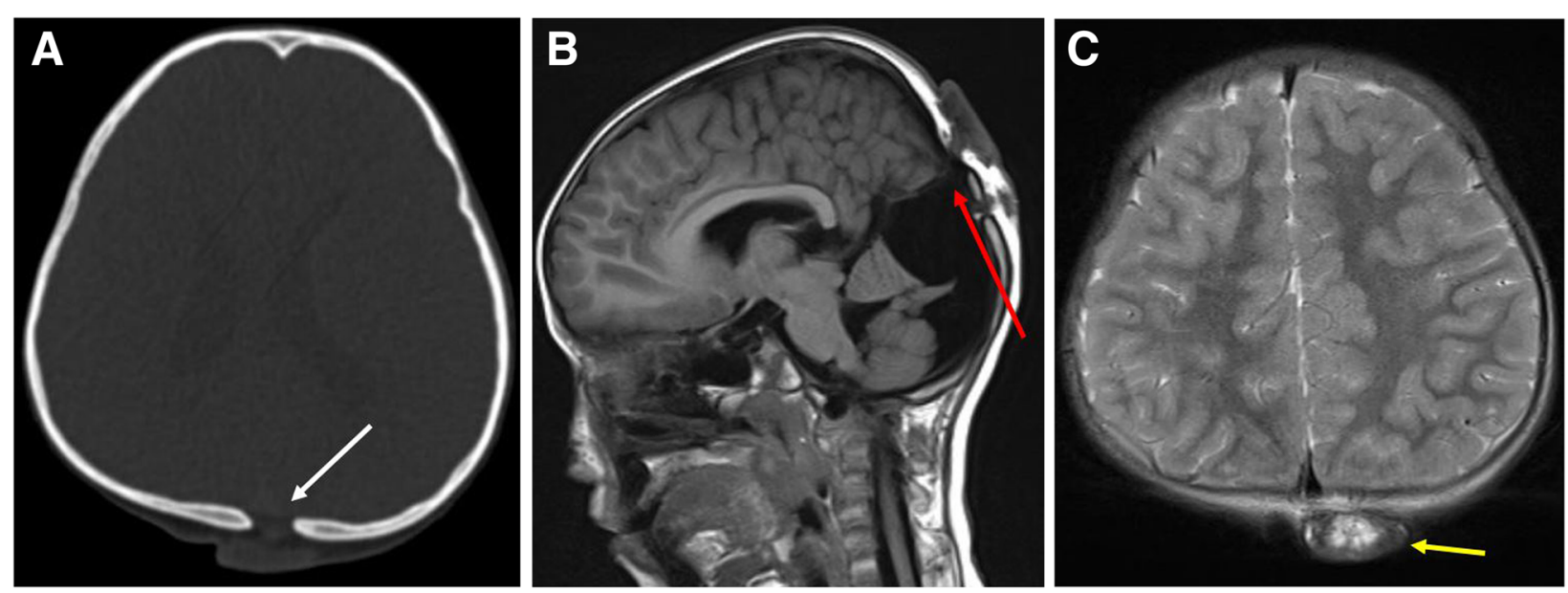

Fig. 7 a Non-contrast axial bone window CT image demonstrates a bony calvarial defect in the occipital bone. b, c Sagittal T1-weighted and axial T2weighted MR images show an occipital scalp swelling with areas of CSF signal intensity within (yellow arrow). It is seen to communicate with posterior fossa cyst through the calvarial defect with associated vertical orientation of straight sinus (red arrow) suggestive of atretic cephalocele 
compressive myelopathy, diastematomyelia in the spinal cord and occipital cephalocele, Chiari malformation type I, and hydrocephalous in the brain have also been observed in patients with KFS. Auditory anomalies and congenital heart diseases are also commonly seen. The visceral findings seen in our patient were atretic occipital cephaloceles, compressive myelopathy, and DandyWalker spectrum. Dandy-Walker malformation is a rare association of KFS. In an extensive literature search, we found only two reports of association of Dandy-Walker malformation with Klippel-Feil syndrome, one of which is reported in antenatal USG and the other was a 6-dayold infant [7, 8]. Dandy-Walker spectrum refers to a group of disorders that do not fulfill criteria for DandyWalker malformation and are characterized by inferior vermian hypoplasia with poorly formed fourth ventricle communicating with posterior fossa cyst. The abnormality rarely causes any specific symptoms and is characterized only in imaging studies.

\section{Conclusion}

The radiologists while evaluating a case of Klippel-Feil syndrome should be aware of the full possible spectrum of associated skeletal and non-skeletal anomalies which guides the treatment planning and has prognostic significance. Therefore, our case report highlights the importance of knowledge of various common and uncommon associations of KFS to avoid missing significant anomalies while reporting.

\section{Abbreviations}

KFS: Klippel-Feil syndrome; CVJ: Craniovertebral junction; NCCT: Non-contrast computed tomography; MRI: Magnetic resonance imaging;

AP: Anteroposterior; CNS: Central nervous system; CSF: Cerebrospinal fluid

\section{Acknowledgements}

Not applicable

\section{Authors' contributions}

MS obtained the patient data, analyzed the images, designed, and drafted the manuscript. MSS and GP revised the manuscript critically for important intellectual content. The authors read and approved the final manuscript.

\section{Funding}

Nil

\section{Availability of data and materials}

Not applicable

Ethics approval and consent to participate

Not applicable

\section{Consent for publication}

Written informed consent was obtained from the parent of the patient for publication of this case report and accompanying images.

Competing interests

The authors declare that they have no competing interests.
Received: 16 November 2020 Accepted: 25 December 2020

Published online: 07 January 2021

References

1. Patel PR, Lauerman WC (1995) Maurice Klippel. Spine 20:2157-2217

2. Wang L, Niu Y, Li X, Hu J, Chen J, Wang S, Wang H (2020) The mutational burden and oligogenic inheritance in Klippel-Feil syndrome. BMC Musculoskelet Disord 21:1-9

3. Samartzis D, Herman J, Lubicky JP, Shen FH (2006) Classification of congenitally fused cervical patterns in Klippel-Feil patients: epidemiology and role in the development of cervical spine-related symptoms. Spine. 31: E798-E804

4. Frikha R (2020) Klippel-Feil syndrome: a review of the literature. Clin Dysmorphol 29:35-37

5. Jovankovičová A, Jakubíková J, Ďurovčíková D (2012) A case of Klippel-Feil syndrome with congenital enlarged eustachian tube. Int J Pediatr Otorhinolaryngol 76:596-600

6. Kadavkolan AS, Bhatia DN, DasGupta B, Bhosale PB (2011) Sprengel's deformity of the shoulder: current perspectives in management. Int J Shoulder Surg 5:1

7. Karaman A, Kahveci H (2011) Klippel-Feil syndrome and Dandy-Walker malformation. Genet Couns 22:411

8. Pirino A, Sotgiu MA, Cosmi E, Montella A, Bandiera P (2019) Association of Klippel-Feil syndrome, Dandy-Walker malformation, spina bifida: a case report. Radiol Case Rep 14:415-418

\section{Publisher's Note}

Springer Nature remains neutral with regard to jurisdictional claims in published maps and institutional affiliations.

\section{Submit your manuscript to a SpringerOpen ${ }^{\circ}$ journal and benefit from:}

- Convenient online submission

- Rigorous peer review

- Open access: articles freely available online

High visibility within the field

- Retaining the copyright to your article

Submit your next manuscript at $>$ springeropen.com 